\title{
ANÁLISE DAS VIBRAÇÕES TRANSMITIDAS AOS TRABALHADORES EM MARCENARIAS NO SUL DO ESPÍRITO SANTO
}

\author{
Nilton César Fiedler' ${ }^{1}$, Rafael Tonetto Alves², Pompeu Paes Guimarães², Fernando Bonelli Wanderley², \\ Luciano José Minette ${ }^{3}$, Amaury Paulo de Souza ${ }^{4}$
}

(recebido: 5 de janeiro de 2009; aceito: 31 de março de 2010)

\begin{abstract}
RESUMO: Esta pesquisa foi desenvolvida em marcenarias localizadas nos municípios de Jerônimo Monteiro e Alegre, sul do Espírito Santo. O objetivo foi quantificar as vibrações transmitidas ao sistema mão-braço dos trabalhadores pelas máquinas nas atividades de fabricação de móveis. Os dados foram coletados por intermédio de um acelerômetro modelo NK 20 e um medidor de vibração modelo NK 300. A vibração foi medida de acordo com as três coordenadas ortogonais definidas na norma ISO 5349-1 (ISO, 2001) e explicada pelo EU Good Practice Guide HAV, seguindo as orientações da Directive 2002/44/EC da União Européia. Os dados foram submetidos a uma análise de variância no delineamento inteiramente casualizado. As médias das máquinas foram comparadas pelo teste de ScottKnott, a 5\% de probabilidade. A desempenadeira apresentou o maior índice de vibração nas coordenadas ortogonais (região de fadiga) e a lixadeira os menores índices de vibração (região de conforto). A análise estatística foi coerente com a análise qualitativa dos dados.
\end{abstract}

Palavras-chave: Ergonomia, indústria moveleira, sistema mão-braço.

\section{VIBRATIONS TRANSMITTED TO WORKERS IN JOINERIES IN THE SOUTH OF ESPÍRITO SANTO STATE}

\begin{abstract}
This research was developed in joineries in the south of the Espirito Santo State (Brazil) and aimed to quantify the vibrations transmitted to workers system hand-arm by the machines in the activities of furniture production. The data were collected by using an accelerometer model NK 20 and a meter of vibration model NK 300. The vibration was measured in agreement with the three orthogonal coordinates defined in ISO 5349-1 and explained by the ME Good Practice Guide HAV, following the orientations of Directive 2002/44/ EC of the European Union. The data were submitted to variance analysis in the entirely casualized experimental design. The averages of the machines were compared by Scott-Knott's test at 5\% probability. The smoother presented the largest vibration index in the orthogonal coordinates (fatigue area) and the sander the smallest vibration indexes (comfort area). The statistical analysis of the averages showed that a good correlation exists between the classification models and the one of analysis of the vibration indexes.
\end{abstract}

Key words: Ergonomics, furniture industry, hand-arm system.

\section{INTRODUÇÃO}

O ambiente de trabalho é composto por um conjunto de fatores interdependentes, que atuam, direta ou indiretamente, na qualidade de vida dos trabalhadores e nos resultados do próprio trabalho. Segundo Iida (2005), uma grande fonte de tensão no trabalho são as condições ambientais desfavoráveis, como excesso de calor, ruídos e vibrações. Esses fatores, segundo Fiedler et al. (2006), causam desconforto, aumentam o risco de acidentes e podem provocar danos consideráveis a saúde.
O ser humano está muitas vezes exposto às vibrações mecânicas que afetam o conforto, a saúde e a segurança, bem como a eficiência do seu desempenho na atividade de fabricação de móveis. A exposição à vibração pode provocar lesões irreversíveis e incapacidades.

Iida (2005) define vibração como sendo qualquer movimento que o corpo executa em torno de um ponto fixo. Esse movimento pode ser regular, do tipo senoidal, ou irregular, quando não segue nenhum padrão determinado. A vibração é um agente nocivo presente em várias atividades do cotidiano do trabalhador.

\footnotetext{
${ }^{1}$ Engenheiro Florestal, Professor Dr. em Ciência Florestal - Departamento de Engenharia Florestal - Centro de Ciências Agrárias Universidade Federal do Espírito Santo - Cx. P. 16 - 29500-000 - Alegre, ES - fiedler@pq.cnpq.br

${ }^{2}$ Engenheiro Florestal, Mestrando do Programa de Pós Graduação em Ciências Florestais - Departamento de Engenharia Florestal Centro de Ciências Agrárias - Universidade Federal do Espírito Santo - Cx. P. 16 - 29500-000 - Alegre, ES - rafatonetto@ gmail.com, pompeupaes@yahoo.com.br, fernandobwef@gmail.com

${ }^{3}$ Engenheiro Florestal, Professor Dr. em Ciência Florestal - Departamento de Engenharia Elétrica e de Produção - Universidade Federal de Viçosa 36571-000 - Viçosa, MG - minette@ufv.br

${ }^{4}$ Engenheiro Florestal, Professor Dr. em Ciência Florestal - Departamento de Engenharia Florestal - Universidade Federal de Viçosa 36571-000 - Viçosa, MG - amaurysouza@ufv.br
} 
As vibrações são particularmente danosas ao organismo nas frequências mais baixas, de 1 a $80 \mathrm{Hertz}$ $(\mathrm{Hz})$. Elas podem provocar lesões nos ossos, juntas e tendões. No sentido longitudinal do corpo (eixo z, dos pés à cabeça), o corpo humano é mais sensível na faixa de 4 a $8 \mathrm{~Hz}$, enquanto no sentido transversal (eixo x e y, perpendiculares ao eixo z) o organismo é mais sensível na faixa de 1 a $2 \mathrm{~Hz}$. As frequências intermediárias, de 30 a 200 $\mathrm{Hz}$, provocam doenças cardiovasculares e nas frequências altas, acima de $300 \mathrm{~Hz}$, o sintoma é de dores agudas e distúrbios neurovasculares.

As vibrações transmitidas ao sistema mão-braço (VMB) são "vibrações mecânicas que, quando transmitidas ao sistema mão-braço, implicam riscos para a saúde e para a segurança dos trabalhadores, em especial perturbações vasculares, lesões osteoarticulares, ou perturbações neurológicas ou musculares" (NUNES, 2005).

Uma das doenças reconhecidas como resultante da exposição prolongada da mão a vibração e impactos repetidos é a síndrome dos dedos brancos. Vários estudos científicos têm sido feitos com o objetivo de determinar a relação da dose-resposta para esta síndrome. Essa doença, também conhecida por síndrome de vibração ou de Raynaud, é causada pelo espasmo das artérias digitais (fenômeno de Raynaud), o que limita o fluxo sanguíneo nos dedos. Como consequiência, os dedos ficam com coloração esbranquiçada e frios. Um dos fatores agravantes dessa doença é a exposição prolongada ao frio. Pensa-se que o hábito de fumar e o stress são, também, fatores que potencializam o seu surgimento. Os sintomas e sinais incluem picadas e dormência dos dedos, formigamento e entorpecimento, espasmos dolorosos da mão, branqueamento de um ou mais dedos e, por vezes, perda de sensação e de controle nos dedos e mãos.

No Brasil, essa síndrome tem recebido destaque, dado que o diagnóstico médico ainda a confunde com a síndrome do túnel do carpo, o que mascara as estatísticas sobre a doença. Uma Lesão por Esforço Repetitivo (LER) ou Distúrbio Osteomuscular Relacionado ao Trabalho (DORT) mal diagnosticada pode encobrir uma síndrome da vibração localizada. Aliados ao fato de que o clima tropical brasileiro atua como agente inibidor do aparecimento da doença, ao contrário dos países de clima frio.

A vibração pode danificar máquinas e estruturas e, por isto, deve ser controlada e/ou isolada. Da mesma forma, observa-se que o corpo humano também pode ser afetado, daí a necessidade de medir e estabelecer limites para a vibração, como agente de controle de insalubridade no trabalho. Na prática, é muito difícil evitar a vibração. Geralmente, ela ocorre por causa dos efeitos dinâmicos de tolerâncias de fabricação, folgas, contatos, o atrito entre peças de uma máquina e, ainda, decorrente das forças desequilibradas de componentes rotativos e de movimentos alternados. É comum verificar que vibrações insignificantes potencializem as frequências de outras peças da estrutura, fazendo com que estas sejam ampliadas, transformando-se em vibrações e ruídos indesejados.

Historicamente, o termo ergonomia é fundamentado no conceito de que o trabalho, ferramenta e postos de trabalho sejam adaptados e ajustados aos trabalhadores, e não o inverso. No entanto, se nenhuma tentativa for feita para amortecer e/ou isolar a fonte de vibração, o design ergonômico transfere vibração mais eficientemente às mãos.

Nesta pesquisa, objetivou-se quantificar as vibrações transmitidas ao sistema mão-braço dos trabalhadores pelas máquinas nas atividades de fabricação de móveis em marcenarias no sul do Espírito Santo.

\section{MATERIAL E MÉTODOS}

\subsection{Região de estudo}

O estudo foi desenvolvido em 3 marcenarias, nos municípios de Jerônimo Monteiro e Alegre, localizados na região sul do Espírito Santo, entre os meses de agosto de 2007 e julho de 2008. As empresas foram escolhidas em razão do fluxo contínuo de produção.

\subsection{População e amostragem}

A avaliação referente à análise de vibração foi realizada por um estudo de caso. Foram escolhidas as principais máquinas envolvidas no processo de fabricação de móveis, a saber: serra circular, desempenadeira, traçador, desengrossadeira, lixadeira e a tupia, conforme descritas na Tabela 1.

Os dados de vibração foram coletados durante a jornada normal de trabalho por intermédio de um acelerômetro modelo NK 20 e um medidor de vibração NK 300 da marca TEKNIKAO (Figura 1).

Para a coleta, a vibração foi medida de acordo com as três coordenadas ortogonais definidas na norma ISO 5349-1 (ISO, 2001) (eixo "xx" - por meio da palma da mão; eixo "yy" - por meio dos nós dos dedos; eixo "zz" - paralelo aos ossos do braço), como demonstrado na Figura 2. 
Tabela 1 - Descrição técnica das máquinas avaliadas.

Table 1 - Technical description of the analyzed machines.

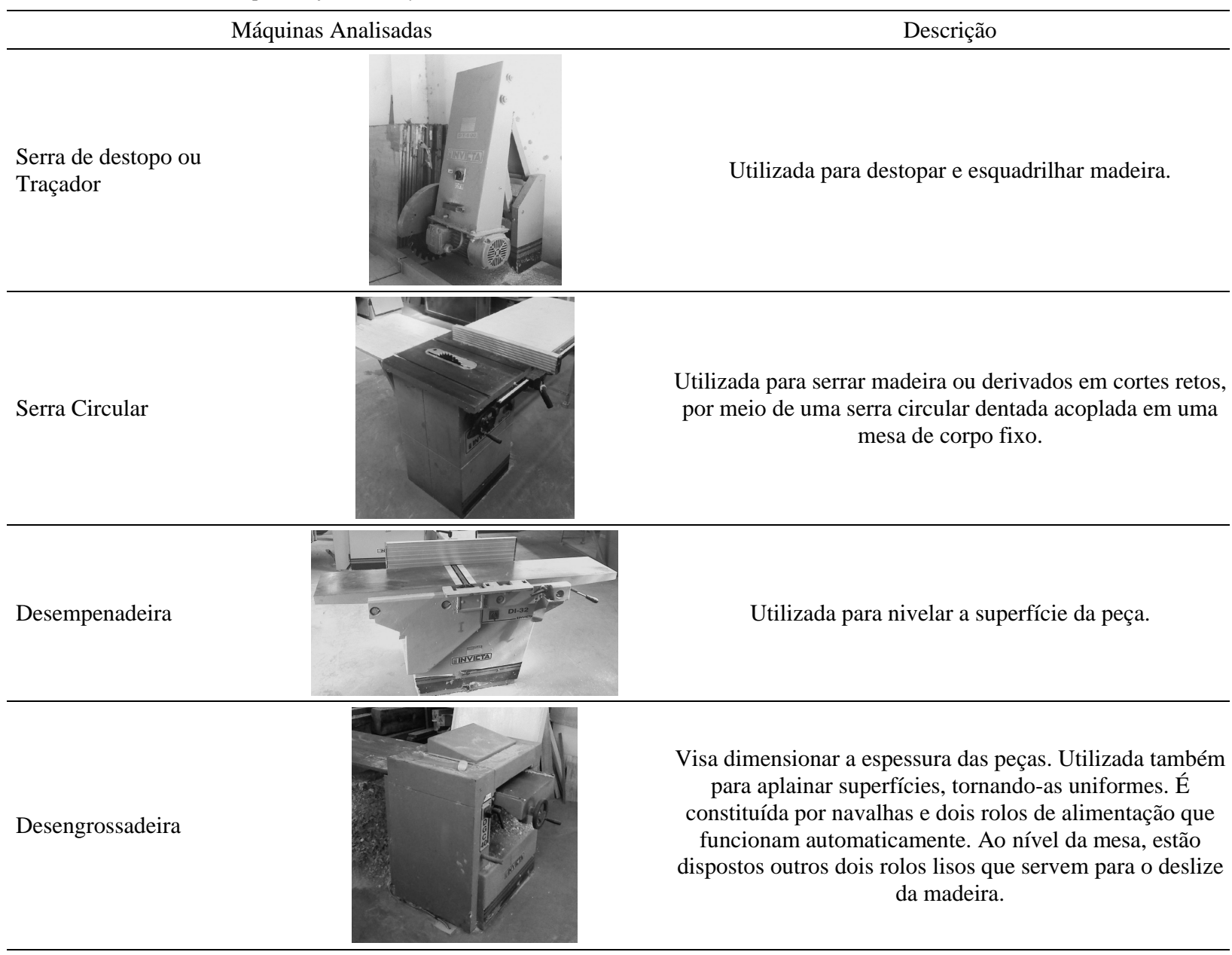

\begin{tabular}{|c|c|}
\hline Serra de fita & $\begin{array}{l}\text { Possui uma versatilidade de trabalho muito grande, podendc } \\
\text { realizar quaisquer tipos de cortes retos ou irregulares, tais } \\
\text { como, círculos, ondulações, etc. Também podemos utilizá- } \\
\text { la para o corte de materiais muito espessos, difíceis de } \\
\text { serem cortados na serra-circular. }\end{array}$ \\
\hline Lixadeira de cinta & $\begin{array}{c}\text { Acabamentos de superfícies planas ou curvas. Elimina } \\
\text { asperezas para que a peça possa receber o acabamento final. } \\
\text { Compõe-se de duas colunas ligadas entre si por uma cinta } \\
\text { de lixa, entre as quais existe uma mesa fixa onde é apoiada } \\
\text { a peça de madeira. }\end{array}$ \\
\hline
\end{tabular}

Cerne, Lavras, v. 16, n. 2, p. 235-242, abr./jun. 2010 


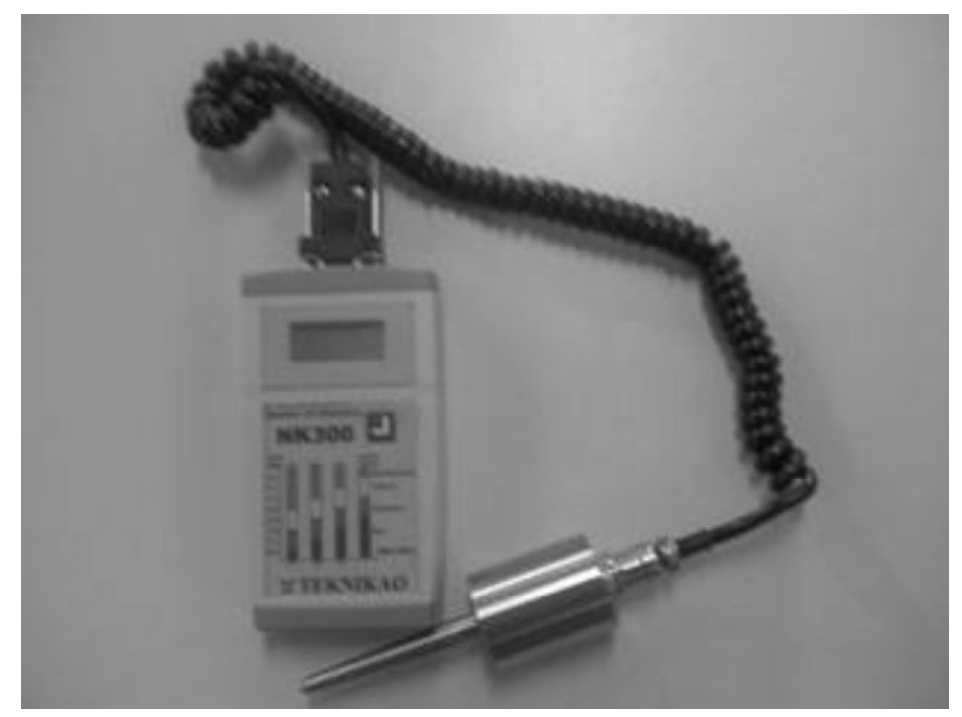

Figura 1 - Acelerômetro e medidor de vibração.

Figure 1-Accelerometer and a meter of vibration.
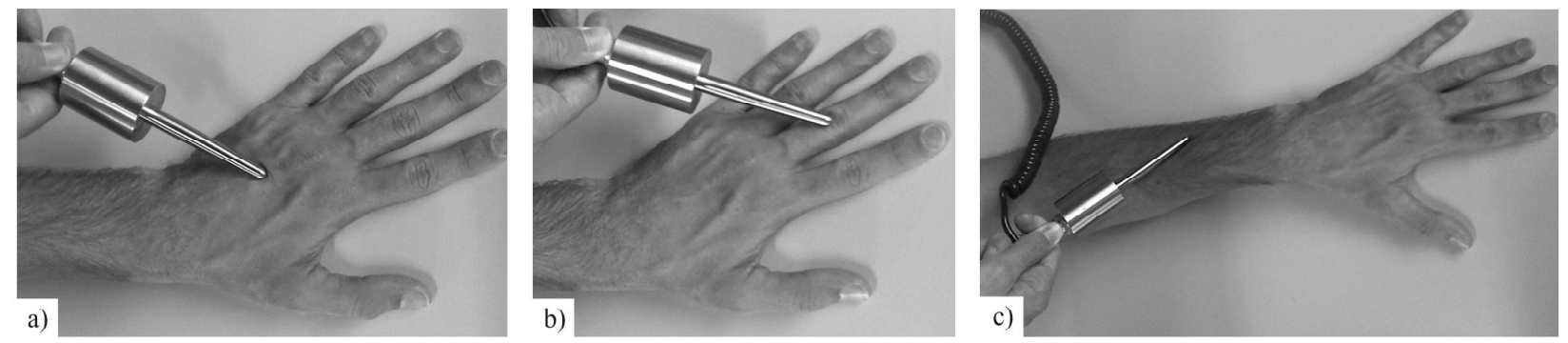

Figura 2 - Coordenadas ortogonais ISO 5349-1 (A: eixo xx; B: eixo yy e C: eixo zz).

Figure 2-Ortogonal coordinates ISO 5349-1 (A: axis xx; B: axis yy and C: axis zz).

Inicialmente, foi feito um estudo piloto para definir o tamanho mínimo da amostra de dados a serem utilizados na pesquisa. Esses dados foram analisados por meio da seguinte expressão, proposta por Conaw (1977), citado por Minette (1996):

$$
n \geq \frac{t^{2} * s^{2}}{e^{2}}
$$

Em que: $\mathrm{n}=$ número de amostras ou pessoas necessárias; $\mathrm{t}=$ valor tabelado a $5 \%$ de probabilidade (distribuição $\mathrm{t}$ de Student); s = desvio-padrão da amostra; e = erro admissível a $5 \%$.

A avaliação do nível de exposição baseia-se no cálculo do valor da exposição diária para um período de referência de 8 horas $(A(8))$.

Cerne, Lavras, v. 16, n. 2, p. 235-242, abr./jun. 2010
Por meio da ISO 2631 de 1978, têm-se os critérios de severidade para adequação dos parâmetros de classificação da vibração mão-braço. Os parâmetros são divididos nos eixos XY e Z; referentes à palma da mão, nós dos dedos e braço, respectivamente.

Para o cálculo da vibração nos eixos XY, utilizou-se a seguinte expressão, definidas na norma ISO 5349-1 (ISO, 2001) e explicada pela EU Good Practice Guide HAV, seguindo as orientações da Directive 2002/44/EC da União Européia:

$$
A(8)=\sqrt{a_{h w x}^{2}+a_{h w y}^{2}}
$$

Em que $a_{\text {hwx }}, a_{\text {hwy }}$ são os valores eficazes (coletados) da aceleração ponderada em frequência, para a palma da mão e nós dos dedos, respectivamente. 
Os valores a considerar para avaliação do risco são mostrados na Tabela 2.

Caso os equipamentos de trabalho sejam usados com ambas as mãos, deve-se medir a vibração em cada uma delas, sendo considerado como valor de exposição o valor mais elevado. No entanto, o valor obtido para a outra mão deve ser também referido. A gestão do risco da exposição a vibrações mecânicas deve seguir o princípio geral da prevenção em segurança, higiene e saúde no trabalho: eliminar o risco na fonte ou reduzi-lo ao mínimo. A eliminação do risco é sempre o meio mais eficaz, devendo por isso ser a primeira opção de controle da vibração a ser considerada. As ações a serem empreendidas dependem, no entanto, do nível de vibração determinado.

A metodologia e os aparelhos utilizados na avaliação do risco de exposição à vibração mão-braço, por medição, seguiu a normatização preconizada pelas partes 1 e 2 da norma ISO 5349 (ISO, 2001).

\subsection{Procedimentos estatísticos}

Para os procedimentos estatísticos, as vibrações coletadas em cada eixo ortogonal foram unidas, seguindo as orientações da ISO 5349-1 (ISO, 2001) e EU Good Practice Guide HAV, utilizando a fórmula proposta por Nunes (2005):

$$
A(8)=\sqrt{a_{h w x}^{2}+a_{h w y}^{2}+a_{h w z}^{2}}
$$

Em que $a_{h w x}, a_{h w y}, a_{h w z}$ são os valores eficazes da aceleração ponderada em frequência, determinados segundo as coordenadas ortogonais, definidas na norma ISO 5349-1 (ISO, 2001) (eixo "xx" - por meio da palma da mão; eixo "yy" - por meio dos nós dos dedos; eixo "zz" - paralelo aos ossos do braço).

Os resultados obtidos foram submetidos a uma análise de variância no delineamento inteiramente casualizado. As médias das máquinas foram comparadas pelo teste de Scott-Knott, a 5\% de probabilidade, quando estas apresentaram diferenças estatísticas significativas.

\section{RESULTADOS E DISCUSSÃO}

\subsection{Classificação das máquinas}

O número mínimo de amostras definido pelo estudo piloto foram de 08 amostras para o traçador (coletados 21 amostras); 29 amostras para a desempenadeira (coletados 40 amostras); 05 amostras para serra fita (coletados 32 amostras); 25 amostras para serra circular (coletados 40 amostras); 15 amostras para a lixadeira (coletados 30 amostras); e 20 amostras para a desengrossadeira (coletados 43 amostras).

Na Tabela 3, observam-se os resultados da análise de vibração nas referidas coordenadas, com a média e a classificação.

Quando o limite do índice de conforto é atingido, o trabalhador já começa a sofrer danos e, quando ultrapassado até os limites de fadiga, o trabalho deve ser reorganizado. Em todas as máquinas amostradas as coordenadas XY apresentaram índices médios acima do limite de conforto, sendo classificados como fadiga.

Assim, sempre que o nível de vibração, na exposição à vibração mão-braço ultrapassar o índice do limite de conforto, referido anteriormente, a entidade patronal deve estabelecer e implementar um programa de medidas técnicas e/ou organizacionais destinadas a reduzir essa exposição para um valor inferior aos valores de conforto; pois a partir deste ponto o trabalhador já está exposto a riscos para sua saúde. Os trabalhos acima dos índices de fadiga não são recomendados e, quando atingidos os níveis de exposição, o trabalho deve ser reorganizado para a redução dos referidos índices.

Justamente a mão e os dedos são as partes que estão sob exposição direta, ou pelo contato com a madeira que é trabalhada, ou pelo contato direto com a máquina.

O traçador e a desempenadeira foram as únicas máquinas que apresentaram índices acima do limite de conforto na coordenada $\mathrm{Z}$, sendo as atividades classificadas como fadiga. As demais máquinas apresentaram índices dentro dos limites de conforto.

Tabela 2 - Guia para a avaliação da exposição humana a vibração (ISO 5349-1, 2001).

Table 2 - Guidelines for assessing human exposure to vibration (ISO 5349-1, 2001).

\begin{tabular}{lcc}
\hline Critérios de Severidade & Aceleração $(\mathrm{X}, \mathrm{Y})$ Horizontal $\left(\mathrm{m} / \mathrm{s}^{2}\right)$ & Aceleração $(\mathrm{Z})$ Vertical $\left(\mathrm{m} / \mathrm{s}^{2}\right)$ \\
\hline Exposição & 0,448 & 0,630 \\
Fadiga & 0,224 & 0,315 \\
Conforto & 0,071 & 0,100 \\
\hline
\end{tabular}


Tabela 3 - Exposição média a vibração no sistema mão-braço que os operadores estão submetidos.

Table 3 - Average exposure to vibration in hand-arm to which operators are submitted.

\begin{tabular}{lcccc}
\hline Máquinas & Coordenadas & Médias $\left(\mathrm{m} / \mathrm{s}^{2}\right)$ & Desvio Padrão & Classificação \\
\hline \multirow{2}{*}{ Desempenadeira } & $\mathrm{XY}$ & 0,160 & 0,051 & Fadiga \\
& $\mathrm{Z}$ & 0,119 & 0,024 & Fadiga \\
\hline \multirow{2}{*}{ Desengrossadeira } & $\mathrm{XY}$ & 0,157 & 0,055 & Fadiga \\
& $\mathrm{Z}$ & 0,097 & 0,019 & Conforto \\
\hline \multirow{2}{*}{ Traçador } & $\mathrm{XY}$ & 0,155 & 0,014 & Fadiga \\
& $\mathrm{Z}$ & 0,110 & 0,015 & Fadiga \\
\hline \multirow{2}{*}{ Serra circular } & $\mathrm{XY}$ & 0,144 & 0,025 & Fadiga \\
& $\mathrm{Z}$ & 0,100 & 0,029 & Conforto \\
\hline \multirow{2}{*}{ Lixadeira } & $\mathrm{XY}$ & 0,165 & 0,013 & Fadiga \\
& $\mathrm{Z}$ & 0,085 & 0,014 & Conforto \\
\hline \multirow{2}{*}{ Serra fira } & $\mathrm{XY}$ & 0,103 & 0,007 & Fadiga \\
& $\mathrm{Z}$ & 0,077 & 0,007 & Conforto \\
\hline
\end{tabular}

A desempenadeira foi a máquina que apresentou o maior índice de vibração nas coordenadas ortogonais, e a lixadeira foi a máquina que apresentou os menores índices de vibração.

O estudo de vibração no sistema mão-braço ainda é incipiente no Brasil, o que gera grande dificuldade em encontrar metodologias e índices para referências, bem como para discussão e análise.

Como os índices de vibração nas coordenadas XY foram classificados como fadiga em todas as máquinas, há a necessidade de se avaliar a melhor forma de atuação nas atividades, porém, medidas de controle direto se tornam dificultadas pela necessidade de destreza manipulativa do operador durante o trabalho, justamente a característica que é mais afetada com a exposição contínua a vibração.

Assim, o melhor modo de atenuar essa exposição está na adoção de algumas medidas organizacionais, também designadas de controle administrativo. São medidas que incidem essencialmente na duração de exposição às vibrações dos trabalhadores. A melhor solução para gerir o risco é sempre aquela que não depende do comportamento do trabalhador.

Entre as medidas mais comumente usadas salientase: a aquisição de máquinas com projetos adequados quanto aos limites de vibração; o revezamento dos trabalhadores entre postos de trabalho com diferentes níveis de solicitação a vibrações; a concepção de ciclo de trabalho-repouso que previna uma exposição longa à vibração; e, a escolha de métodos de trabalho alternativos que permitam reduzir a exposição a vibrações mecânicas.

\subsection{Análise estatística}

As médias dos índices de vibração para análise de variância acompanhados do desvio padrão são mostrados na Tabela 4.

Os resultados da análise de variância para o delineamento inteiramente casualizado dos índices de vibração são apresentados na Tabela 5. Foram verificadas diferenças significativas entre as máquinas, realizando o teste de Scott Knott, ambos a 5\% de significância.

De acordo com os resultados apresentados na Tabela 5, foi necessária a utilização de um teste de comparações de médias para avaliação das similaridades e das diferenças dos índices de vibração existentes entre as máquinas. Os resultados do teste de Scott Knott para os índices de vibração nas empresas são apresentados na Tabela 6.

O teste de comparações de médias serviu para mostrar diferenças estatísticas entre as máquinas, onde as máquinas seguidas de mesma letra não apresentaram diferença estatística dos índices de vibração. A desempenadeira que apresentou o maior índice de vibração diferenciou-se das demais máquinas; o traçador, a desengrossadeira e a serra circular, não diferiram estatisticamente entre si. A serra fita apresentou o menor índice de vibração entre as máquinas.

A análise estatística das médias mostrou que existe uma boa correlação entre os modelos de classificação e o de análise dos índices de vibração.

Cerne, Lavras, v. 16, n. 2, p. 235-242, abr./jun. 2010 
Tabela 4 - Índices de vibração para análise de variância.

Table 4 - Vibration indexes for variance analysis.

\begin{tabular}{lcc}
\hline Máquina & Médias $\left(\mathrm{m} / \mathrm{s}^{2}\right)$ & Desvio Padrão \\
\hline Desempenadeira & 0,2137349 & 0,0338971 \\
Desengrossadeira & 0,1842909 & 0,0541663 \\
Traçador & 0,1897613 & 0,01285337 \\
Serra Circular & 0,1752209 & 0,0341904 \\
Lixadeira & 0,1499628 & 0,0141857 \\
Serra Fita & 0,1273021 & 0,0062521 \\
\hline
\end{tabular}

Tabela 5 - Resultados da análise de variância para os índices de vibração.

Table 5 - Results of the variance analysis for the vibration indexes.

\begin{tabular}{lcccc}
\hline FV & G.L. & SQ & QM & F \\
\hline Máquina & 5 & 0,08857443 & 0,01771489 & $16,541^{*}$ \\
Resíduo & 129 & 0,1381583 & 0,001070994 & \\
\hline Total & 134 & 0,22673273 & 0,018785884 & \\
\hline
\end{tabular}

* significativo ao nível de 5\%

Tabela 6 - Resultados do teste de comparações de médias de Scott-Knott para os índices de vibração gerada pelas máquinas estudadas.

Table 6-Results of the test of comparisons of averages of Scott Knott for the vibration indexes generated by the studied machines.

\begin{tabular}{lllll}
\hline Máquina & Médias $\left(\mathrm{m} / \mathrm{s}^{2}\right)$ & & \\
\hline Desempenadeira & 0,2137349 & $\mathrm{a}$ & & \\
Desengrossadeira & 0,1842909 & $\mathrm{~b}$ & \\
Traçador & 0,1897613 & $\mathrm{~b}$ & \\
Serra Circular & 0,1752209 & $\mathrm{~b}$ & & \\
Lixadeira & 0,1499628 & & $\mathrm{c}$ & \\
Serra Fita & 0,1273022 & & $\mathrm{~d}$ & \\
\hline
\end{tabular}

\section{CONCLUSÕES}

Todas as máquinas apresentaram classificação de fadiga nas coordenadas XY.

A desempenadeira foi a máquina que apresentou os maiores índices de vibração para todas as coordenadas ortogonais.

A análise estatística mostrou que não houve diferença estatística entre traçador, desengrossadeira e a serra circular.

A serra fita foi a máquina que apresentou os menores índices de vibração nas coordenadas ortogonais.
Em razão da classificação de fadiga para mão e dedos em todas as máquinas, há a necessidade de se adotar medidas organizacionais, como referido, para minimizar os efeitos da exposição sobre os trabalhadores.

\section{REFERÊNCIAS BIBLIOGRÁFICAS}

FIEDLER, N. C.; RODRIGUES, T. O.; MEDEIROS, M. B. Avaliação das condições de trabalho, treinamento, saúde e segurança de brigadistas de combate a incêndios florestais em unidades de conservação do Distrito Federal. Revista Árvore, Viçosa, v. 30, n. 1, p. 55-63, 2006. 
IIDA, I. Ergonomia: projeto e produção. 2. ed. rev. e ampl. São Paulo: E. Blucher, 2005. 630 p.

ISO guide for the evaluation of human exposure to wholebody vibration: ISO 2631. Geneva: ISO, 1978. 15 p.

Minette, L. J. Análise de fatores operacionais e ergonômicos na operação de corte florestal com motosserra.
1996. 211 p. Tese (Doutorado em Ciência Florestal) Universidade Federal de Viçosa, Viçosa, 1996.

NUNES, I. L. A importância da avaliação de factores de risco ocupacionais em políticas de aquisição de equipamentos: o caso da exposição à vibração. In: JORNADAS TÉCNICAS DE ENGENHARIA LOGÍSTICA MILITAR - O CICLO DE VIDA DOS MATERIAIS, 3., 2005, Paço de Arcos. Anais... Paço de Arcos, 2005. CD-ROM.

Cerne, Lavras, v. 16, n. 2, p. 235-242, abr./jun. 2010 